\title{
A chromosomal region surrounding the ompD porin gene marks a genetic difference between Salmonella typhi and the majority of Salmonella serovars
}

\author{
Carlos A. Santiviago, Cecilia S. Toro,† Sergio A. Bucarey \\ and Guido C. Mora
}

\author{
Author for correspondence: Guido C. Mora. Tel: +562 6862849. Fax: +56 22225515 \\ e-mail: gmora@genes.bio.puc.cl
}

Laboratorio de Microbiología,

Departamento de Genética Molecular y Microbiología,

Facultad de Ciencias Biológicas, Pontificia Universidad Católica de Chile, Alameda no. 340, Santiago, Chile
In this work it is shown that the majority of Salmonella serovars most frequently associated with the systemic infection of vertebrate hosts produce a major outer-membrane porin, OmpD. However, OmpD is absent from the outer-membrane protein profiles of Salmonella typhi strain Ty2 and 26 clinical isolates of S. typhi examined by SDS-PAGE. To determine whether the ompD gene is present in $S$. typhi, primers internal to the ompD coding sequence were used to amplify the gene by PCR. With the exception of S. typhi strains, the ompD gene was amplified from the genomes of all Salmonella serovars tested. Consistently, a specific ompD probe did not hybridize with DNA isolated from the $S$. typhi strains. Taken together, these results demonstrate that $S$. typhi does not produce OmpD due to the absence of the ompD gene. Furthermore, it was investigated whether the deletion of ompD extended to smvA. This gene is adjacent to ompD in the Salmonella typhimurium chromosome and encodes a protein involved in the resistance to methyl viologen, a superoxide-generating agent. Although PCR failed to amplify the smvA gene from the S. typhi strain Ty2 genome, it was possible to amplify it from the chromosome of the clinical strains. On the other hand, hybridization analyses showed that the smvA gene is present in all the S. typhi strains tested. In contrast to the other Salmonella serovars, S. typhi strain Ty2 and the clinical isolates showed sensitivity to methyl viologen, suggesting that smvA gene is inactive in S. typhi. In conclusion, the ompD-smvA region is variable in structure among Salmonella serovars. It is hypothesized that the absence of ompD may suggest a role in host specificity.

Keywords: Salmonella, outer-membrane protein, smvA, genetic variability, host specificity

\section{INTRODUCTION}

Salmonella typhi causes the systemic disease typhoid fever only in its human host. The closely related Salmonella typhimurium causes a typhoid-like systemic disease in the mouse, but most frequently causes only a self-limited gastroenteritis in the human host. Several

\footnotetext{
+Present address: Programa de Microbiología, Instituto de Ciencias Biomédicas, Universidad de Chile, Independencia no. 127, Santiago, Chile. Abbreviation: OMP, outer-membrane protein.
}

genetic variations between $S$. typhi and S. typhimurium have been suggested to contribute to their respective host specificity and infectivity. The relative order of genes on the S. typhimurium and S. typhi chromosomes is different, as is the relative stability of their genomes. In addition, the $S$. typhi genome is considered to be fluid because different chromosome structures are found among clinical isolates (Echeita \& Usera, 1998; Liu \& Sanderson, 1996). Many of these genomic rearrangements are thought to be due to homologous recombination events between pairs of the seven rRNA $(r r n)$ operons. Thus, it has been postulated that genomic 
rearrangements, rather than mutations, are responsible for producing the ribotype heterogeneity in S. typhi (Ng \& Sanderson, 1999). In contrast, no rrn-mediated rearrangements have been detected among wild-type strains of S. typhimurium (Liu \& Sanderson, 1995b; Liu $\&$ Sanderson, 1996) in spite of the high frequency of rearrangements observed for the S. typhimurium strain LT2 when grown in culture (Anderson \& Roth, 1981). S. typhi appears to be exceptional among enteric bacteria because of its significant genome structure plasticity (Krawiec \& Riley, 1990; Liu \& Sanderson, 1996).

Because the sequences of conserved genes between $S$. typhi and S. typhimurium are nearly identical, they are said to be homeologous (Zahrt \& Maloy, 1997; Zahrt et al., 1994). However, it is unlikely that the minor fraction $(1-2 \%)$ of nucleotide base pair differences between genes conserved in these two serovars contributes significantly to their remarkably different host specificities. Rather, the striking differences in S. typhi and $S$. typhimurium host specificity is most likely due to the gross rearrangements one observes when comparing their structures at a more macroscopic level. The substitutions and insertions between $S$. typhi and $S$. typhimurium genomes range in size from islands comprised of more than 100 genes to smaller substitutions corresponding to single ORFs (McClelland \& Wilson, 1998). In addition, about $20 \%$ of the S. typhi genome is not present in S. typhimurium and vice versa (Lan \& Reeves, 1996). Therefore, it is possible that as many as 900 S. typhi genes could be serovar-specific, a subset of which is likely to be involved in determining host range (Bäumler et al., 1998).

In this work, we describe one of the smaller interserovar differences in genome structure between $S$. typhi and $S$. typhimurium. The region of the S. typhimurium genome including $s m v A$, ompD and $y d d G$ genes is different in $S$. typhi. These genes encode proteins that confer resistance to methyl viologen, to an abundant outer-membrane porin and to a putative transmembrane permease, respectively. The primary structure of the region containing these functions is genetically variable among Salmonella serovars.

\section{METHODS}

Bacterial strains and growth conditions. The Salmonella strains used in this study are listed in Table 1. The $S$. typhi clinical strains (STH) were obtained from the Infectious Diseases Hospital (Hospital Lucio Córdova) in Santiago, Chile. Bacteria were grown routinely at $37^{\circ} \mathrm{C}$ in Luria-Bertani broth (LB; Bacto tryptone, $10 \mathrm{~g} \mathrm{l}^{-1}$; Bacto yeast extract, $5 \mathrm{~g}$ $\mathrm{l}^{-1} ; \mathrm{NaCl}, 5 \mathrm{~g} \mathrm{l}^{-1}$ ) and aerated by shaking. When required, LB was supplemented with ampicillin $\left(100 \mathrm{mg} \mathrm{l}^{-1}\right)$ and tetracycline $\left(10 \mathrm{mg} \mathrm{l}^{-1}\right)$. Media were solidified by the addition of $\operatorname{agar}\left(15 \mathrm{~g} \mathrm{l}^{-1}\right)$.

Genetic techniques. Transductional crosses were carried out at low m.o.i. using the high-frequency transducing phage P22 HT105/1 int-201 as described by Maloy (1990). A recombinant $S$. typhi Ty 2 ompD ${ }^{+}$strain was obtained by transducing the wild-type ompD gene from $S$. typhimurium LT2 to $S$. typhi strain Ty2. In a first cross, the ompD::Tn10 allele was transduced using phage P22 from S. typhimurium strain MST2944 to $S$. typhi strain Ty2, selecting for tetracycline resistance. In a second cross, the wild-type $o m p D$ allele was transduced from $S$. typhimurium strain LT2 to $S$. typhi strain Ty2 ompD:: Tn10 to produce the S. typhi strain Ty2 ompD $D^{+}$. The latter was obtained by selecting for loss of tetracycline resistance on Bochner plates (Maloy \& Nunn, 1981). Serotypes of the transductants were confirmed by agglutination using specific antisera against $\mathrm{O}$-antigen groups D1 and B.

Preparation of outer-membrane proteins (OMPs). Outermembrane fractions were prepared as described by Lobos \& Mora (1991), based on a modification of the method of Schnaitman (1971). Bacteria were grown overnight at $37^{\circ} \mathrm{C}$ and harvested by centrifugation at $13000 \mathrm{~g}$ for $15 \mathrm{~min}$. Cell pellets were washed and resuspended in $1 \mathrm{ml} 10 \mathrm{mM}$ Tris $/ \mathrm{HCl}$ pH 8.0 buffer. Bacteria were disrupted by sonication (Vibra Cell, Sonics \& Materials) for $100 \mathrm{~s}$ and centrifuged at $8000 \mathrm{~g}$ for $5 \mathrm{~min}$. Supernatants were collected and centrifuged at $13000 \mathrm{~g}$ for $30 \mathrm{~min}$. Supernatants were then discarded and pellets were resuspended in $500 \mu \mathrm{l}$ buffer containing $10 \mathrm{mM}$ Tris $/ \mathrm{HCl} \mathrm{pH} 8.0,10 \mathrm{mM} \mathrm{MgCl}, 2 \%$ Triton X-100. After incubation at $37^{\circ} \mathrm{C}$ for $30 \mathrm{~min}$, mixtures were centrifuged at $13000 \mathrm{~g}$ for $30 \mathrm{~min}$. The insoluble outer-membrane fraction was resuspended in buffer consisting of $100 \mathrm{mM}$ Tris/ $\mathrm{HCl}$ $\mathrm{pH} 8.0,2 \%$ SDS. SDS-PAGE was performed in $12.5 \%$ polyacrylamide slabs as described by Lobos \& Mora (1991).

PCR amplifications. All amplifications were conducted using a Perkin Elmer thermal cycler (GeneAmp PCR System 2400) and Taq polymerase (Gibco-BRL). The final volume in the tubes for amplification was $50 \mu \mathrm{l}$ and consisted of $1 \times$ Taq PCR buffer, $1.5 \mathrm{mM} \mathrm{MgCl}_{2}$, each $\mathrm{dNTP}$ at a concentration of $200 \mu \mathrm{M}, 50 \mathrm{pmol}$ of each primer, $0.1 \mathrm{mg}$ DNA and $1.25 \mathrm{U}$ Taq polymerase. Standard conditions for amplification were 30 cycles at $94^{\circ} \mathrm{C}$ for $1 \mathrm{~min}, 55^{\circ} \mathrm{C}$ for $1 \mathrm{~min}$ and $72{ }^{\circ} \mathrm{C}$ for $2 \mathrm{~min}$, followed by a final extension step at $72{ }^{\circ} \mathrm{C}$ for $5 \mathrm{~min}$. Primers OMPD1 (5'-GAC AAA GAC AAA ACC CGT T-3') and OMPD2 (5'-CGT CCA GCA GGT TGA TTT T-3') were used to amplify a $740 \mathrm{bp}$ internal fragment of the ompD gene (Singh et al., 1996). Primers SMVA1 (5'-CTT AAC CGC CCG CTA TGA T-3') and SMVA2 (5'-GCT GAA CCA CAT CCC TAC C-3') were designed from the reported $s m v A$ sequence (GenBank accession no. D26057) and used to amplify a $940 \mathrm{bp}$ fragment internal to $s m v A$.

Southern hybridizations. Plasmid pNK2883 (Kleckner et al., 1991) was cleaved with BamHI, and the $3 \mathrm{~kb}$ fragment with the tetRA genes was purified, labelled using the Bioprime DNA labelling system (Gibco-BRL) and used as the probe for Tn10. Hybridization probes for ompD and $s m v A$ were generated by PCR using a mixture of dNTPs containing biotin-14-dCTP and purified by using the Concert gel extraction system (Life Technologies). Genomic DNA of Salmonella serotypes was prepared as described by Sambrook et al. (1989), restricted with PstI (Gibco-BRL) and the fragments were separated on a $0.8 \%$ agarose gel. The DNA was then transferred onto a nylon membrane and cross-linked by UV irradiation. Hybridization was performed in solutions without formamide at $65^{\circ} \mathrm{C}$. Two 15 min washes were performed at $65^{\circ} \mathrm{C}$ in a buffer consisting of $0.5 \mathrm{M} \mathrm{Na}_{2} \mathrm{HPO}_{4} \mathrm{pH} 7 \cdot 2,2 \%$ SDS, $1 \mathrm{mM}$ EDTA. Hybridization was detected by using the nonradioactive Photogene nucleic acid detection system (Life Technologies) and XAR-5 Kodak films. 
Table 1. Salmonella strains used in this study

\begin{tabular}{|c|c|c|}
\hline Strain* & Genotype/phenotype & Source $†$ \\
\hline \multicolumn{3}{|l|}{ S. typhi } \\
\hline Ty 2 & Vaccine strain, Vi minus & WHO \\
\hline TYT2015 & Ty2 ompD159::Tn10 & This work \\
\hline TYT2016 & Ty2 ompD $D^{+}$ & This work \\
\hline \multicolumn{3}{|l|}{ S. typhimurium } \\
\hline LT2 & Wild-type & S. Maloy \\
\hline SC1 & LT2 ompD159::Tn10 & This work \\
\hline MST2944 & $\begin{array}{l}\text { ompD159:: Tn10 galE496 his-6165 ilv-452 hspLT6 } \\
\text { hspS29 metE551 xyl-404 rpsL120 fla-66 metA22 } \\
\text { trpC2 H1-b H2-e,n,x nml }\end{array}$ & S. Maloy \\
\hline \multicolumn{3}{|l|}{ Other salmonellae } \\
\hline S. agona SARB 1 & Wild-type & S. Maloy \\
\hline S. anatum SARB 2 & Wild-type & S. Maloy \\
\hline S. brandenburg SARB 3 & Wild-type & S. Maloy \\
\hline S. choleraesuis SARB 4 & Wild-type & S. Maloy \\
\hline S. decatur SARB 8 & Wild-type & S. Maloy \\
\hline S. derby SARB 9 & Wild-type & S. Maloy \\
\hline S. dublin SARB 12 & Wild-type & S. Maloy \\
\hline S. duisberg SARB 15 & Wild-type & S. Maloy \\
\hline S. enteritidis SARB 16 & Wild-type & S. Maloy \\
\hline S. emek SARB 20 & Wild-type & S. Maloy \\
\hline S. gallinarum SARB 21 & Wild-type & S. Maloy \\
\hline S. haifa SARB 22 & Wild-type & S. Maloy \\
\hline S. heidelberg SARB 23 & Wild-type & S. Maloy \\
\hline S. infantis SARB 26 & Wild-type & S. Maloy \\
\hline S. miami SARB 28 & Wild-type & S. Maloy \\
\hline S. muenchen SARB 32 & Wild-type & S. Maloy \\
\hline S. newport SARB 36 & Wild-type & S. Maloy \\
\hline S. panama SARB 39 & Wild-type & S. Maloy \\
\hline S. paratyphi A SARB 42 & Wild-type & S. Maloy \\
\hline S. paratyphi B SARB 43 & Wild-type & S. Maloy \\
\hline S. paratyphi C SARB 48 & Wild-type & S. Maloy \\
\hline S. pullorum SARB 51 & Wild-type & S. Maloy \\
\hline S. reading SARB 53 & Wild-type & S. Maloy \\
\hline S. rubislaw SARB 54 & Wild-type & S. Maloy \\
\hline S. saintpaul SARB 56 & Wild-type & S. Maloy \\
\hline S. schwarzengrund SARB 57 & Wild-type & S. Maloy \\
\hline S. sendai SARB 58 & Wild-type & S. Maloy \\
\hline S. senftenberg SARB 59 & Wild-type & S. Maloy \\
\hline S. stanley SARB 60 & Wild-type & S. Maloy \\
\hline S. thompson SARB 62 & Wild-type & S. Maloy \\
\hline S. typhisuis SARB 70 & Wild-type & S. Maloy \\
\hline S. wien SARB 71 & Wild-type & S. Maloy \\
\hline S. montevideo 3799-98 & Wild-type & ISP \\
\hline
\end{tabular}

*SARB, Salmonella Reference Collection B (Selander et al., 1996).

†S. Maloy, University of Illinois, USA; ISP, Instituto de Salud Pública (Public Health Institute), Santiago, Chile.

Tests for resistance to methyl viologen (paraquat). Each of the Salmonella strains tested was isolated on MacConkeylactose agar, and single colonies were streaked onto LB agar plates containing $0.5 \mathrm{mM}$ methyl viologen, an agent that generates superoxide and oxygen radicals (Farr \& Kogoma, 1991). Plates were incubated overnight at $37^{\circ} \mathrm{C}$ and scored for bacterial growth. To determine the minimal dose of methyl viologen required to kill Salmonella strains, a lawn of the strain being tested was seeded on LB agar plates and $10 \mu \mathrm{l}$ of serial dilutions of methyl viologen stock were added. Plates were incubated overnight at $37^{\circ} \mathrm{C}$ and scored for bacterial growth. 


\section{RESULTS}

\section{S. typhi does not produce the OmpD porin}

Previously, we have reported that $S$. typhi strain Ty2 does not produce the OmpD porin (Calderón et al., 1984). In contrast, OmpD is one of the most abundant proteins in the S. typhimurium outer membrane (Lee \& Schnaitman, 1980; Nikaido \& Vaara, 1985; Nikaido, 1996). To investigate the presence of OmpD among the majority of Salmonella serovars most frequently associated with systemic infection of vertebrate hosts, the OMPs of a variety of Salmonella serovars were analysed by SDS-PAGE. Electrophoretic profiles of OMPs produced by Salmonella enteritidis, Salmonella paratyphi A, Salmonella paratyphi B, Salmonella paratyphi C, Salmonella choleraesuis, Salmonella gallinarum, Salmonella pullorum, Salmonella dublin, Salmonella saintpaul, Salmonella muenchen, Salmonella heidelberg and Salmonella montevideo showed that all of these serovars produce a major protein migrating as S. typhimurium OmpD porin (Fig. 1a).

The same protein band was observed when this analysis was extended to include Salmonella agona, Salmonella anatum, Salmonella brandenburg, Salmonella decatur, Salmonella derby, Salmonella duisberg, Salmonella emek, Salmonella haifa, Salmonella infantis, Salmonella miami, Salmonella newport, Salmonella panama, Salmonella reading, Salmonella rubislaw, Salmonella schwarzengrund, Salmonella sendai, Salmonella senftenberg, Salmonella stanley, Salmonella thompson, Salmonella typhisuis and Salmonella wien (data not shown). This protein band disappeared from the SDSPAGE OMP profiles when the ompD::Tn10 allele was transduced from S. typhimurium strain MST2944 to $S$. enteritidis, S. paratyphi A, S. paratyphi B, S. pullorum and $S$. gallinarum recipient strains, by using phage $\mathrm{P} 22$ (Fig. 1b). These data indicate that the protein band corresponds to the OmpD porin in these serovars, making it highly probable that it also represents OmpD in all the Salmonella serovars included in this study. On the other hand, the analysis of OMPs from $S$. typhi strain Ty2 (Fig. 1a) and 26 independent clinical isolates of $S$. typhi (data not shown) indicated that OmpD is not produced by these strains.

The finding that S. typhi strains do not produce OmpD can be explained by one of two alternative hypotheses. $S$. typhi may have an ompD gene that is not expressed, or there may be no homologous ompD gene in S. typhi. Because the current sequence data produced by the $S$. typhi Sequencing Group at the Sanger Center (ftp:// ftp.sanger.ac.uk/pub/pathogens/st/ST.dbs) do not include an OmpD coding sequence, sequence data alone do not allow the distinction between these two hypotheses. Therefore, a combination of genetic and biochemical experiments was designed to determine whether the ompD gene is absent from the S. typhi genome.

\section{PCR and DNA hybridization analyses indicate that the ompD gene is absent from the S. typhi genome}

To determine whether the ompD gene is present in $S$. typhi, we first attempted to amplify a $740 \mathrm{bp}$ fragment internal to ompD using PCR, with ompD-specific oligonucleotide primers (Singh et al., 1996). With these primers, no product was obtained when DNA isolated

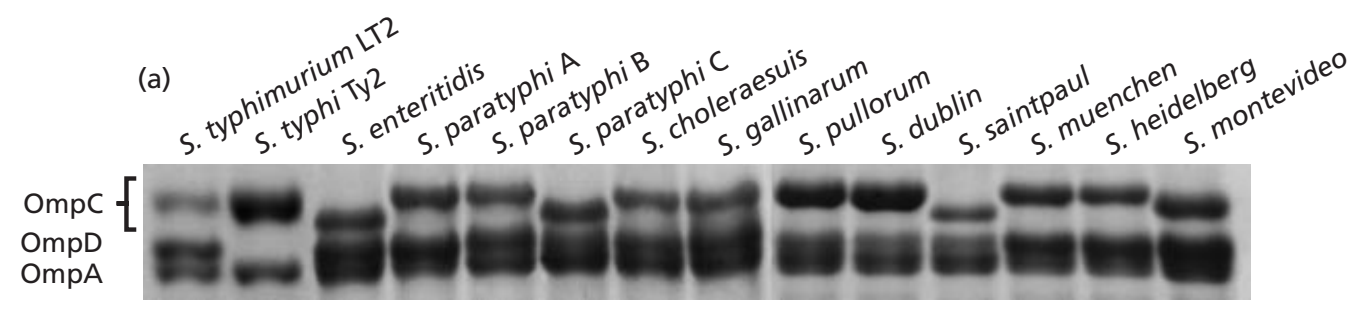

(b)

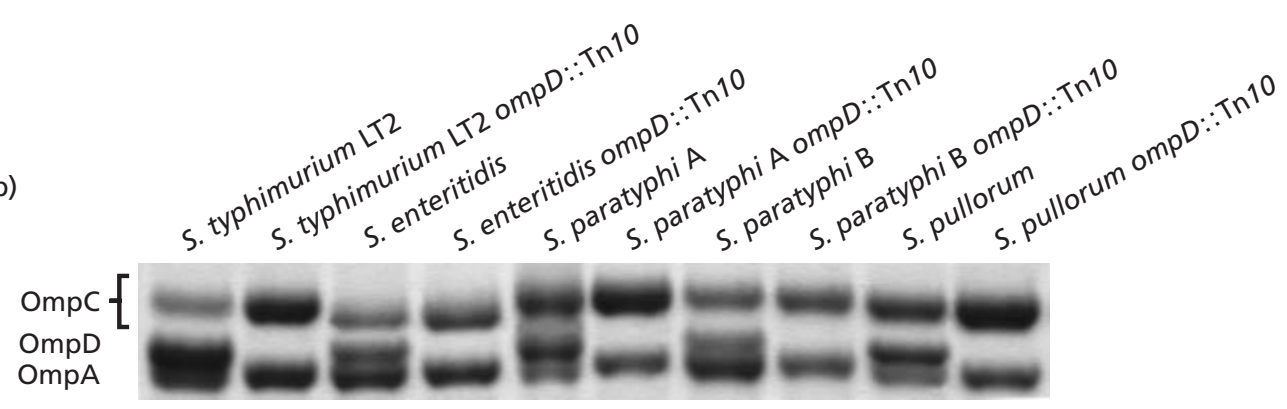

Fig. 1. SDS-PAGE analysis of porins from several Salmonella serovars. OMPs were prepared from bacteria grown aerobically at $37^{\circ} \mathrm{C}$ in LB broth to stationary phase. Protein samples were resolved by electrophoresis through $12.5 \%$ acrylamide and stained with Coomassie blue. Under the bacterial growth conditions used, OmpF porin is not detected in the OMP profiles. 

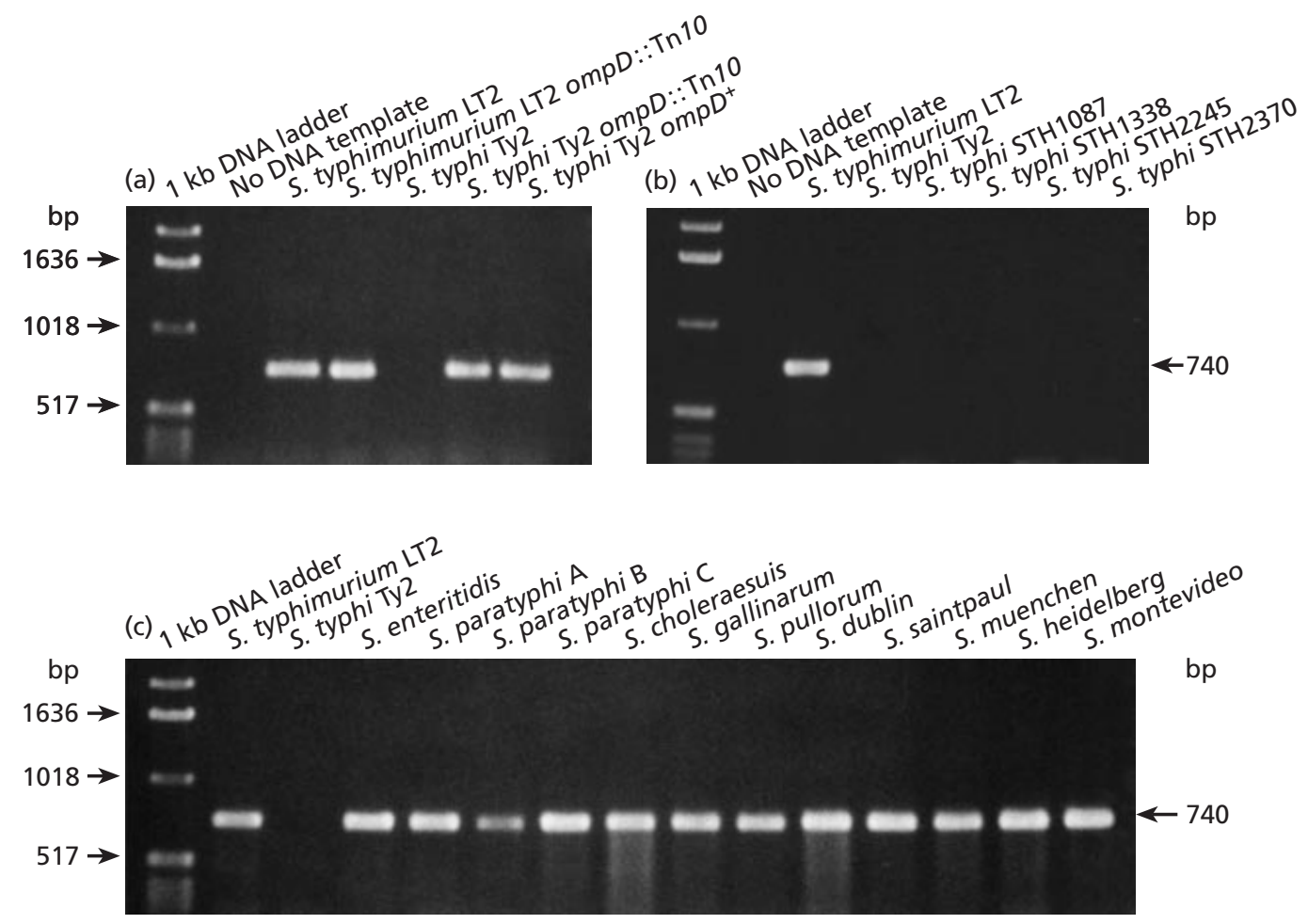

Fig. 2. Amplification of the $o m p D$ gene from several Salmonella serovars. $P C R$ amplifications were performed as described in Methods, using primers internal to ompD. Chromosomal DNA templates were obtained from (a) interspecific hybrid strains between S. typhimurium and S. typhi, (b) S. typhi clinical strains and (c) several Salmonella serovars.

from S. typhi strain Ty2, or DNA isolated from each of 26 independent S. typhi clinical isolates, was used as template (see Fig. $2 \mathrm{~b}$ for example). In contrast, a product of the predicted size was amplified using the same primers and DNA templates prepared from all the other Salmonella serovars tested (Fig. 2c).

Since deficient primer annealing due to low homology could account for the absence of PCR amplification of the $\operatorname{ompD}$ gene, the presence of the ompD gene in $S$. typhi was evaluated directly by DNA hybridization. This method is less dependent on the sequence homologies between the S. typhi and S. typhimurium serovars. Therefore, the 740 bp ompD fragment obtained from $S$. typhimurium was labelled and used to probe Southern blots of S. typhi DNA. The ompD probe hybridized neither with DNA obtained from $S$. typhi strain Ty2, nor with DNA from each of 10 randomly chosen S. typhi clinical isolates (Fig. 3a). In contrast, it did hybridize with a band of approximately $4.4 \mathrm{~kb}$ in DNA samples obtained from S. typhimurium strain LT2 (Fig. 3a). This small band indicates a DNA fragment containing the wild-type ompD gene. The DNAs of several Salmonella serovars were also examined by Southern blot hybridization using the highly specific probe to the ompD gene. Hybridization was observed in three patterns, one of which was identical to that observed using S. typhimurium DNA and included S. brandenburg, S. emek, S. haifa, S. heidelberg, S. infantis, S. miami, S. panama, S. paratyphi A, S. pullorum, S. reading, $S$. schwarzengrund and $S$. wien serovars. The second hybridization pattern revealed a band of approximately $14 \mathrm{~kb}$ in DNA samples obtained from S. agona, $S$. anatum, S. choleraesuis, S. decatur, S. derby, S. dublin, S. duisberg, S. enteritidis, S. gallinarum, S. montevideo, S. muenchen, S. newport, S. paratyphi C, S. rubislaw, S. saintpaul, S. sendai, S. stanley, S. thompson and S. typhisuis serovars. The third pattern of hybridization revealed a band of approximately $11.5 \mathrm{~kb}$, and was only observed in DNA samples obtained from S. paratyphi B (Fig. 3b). These results strongly suggest that the ompD locus is widely distributed among Salmonella serovars, with the exception of S. typhi.

\section{The S. typhimurium ompD gene can be transduced to and expressed in S. typhi}

To understand the genetic variation of the ompD gene between S. typhi and S. typhimurium, we assessed the feasibility of constructing a derivative of $S$. typhi harbouring the $S$. typhimurium ompD gene (S. typhi strain Ty 2 ompD $D^{+}$). Previously, it has been shown that multiple sequence differences in genes conserved between these two serovars present a formidable barrier to the construction of interspecific hybrids. This is because both mismatch repair and the RecBCD recombination pathways inhibit homologous recombination events (Zahrt \& Maloy, 1997; Zahrt et al., 1994). Nonetheless, it is possible to introduce small regions of the $S$. 

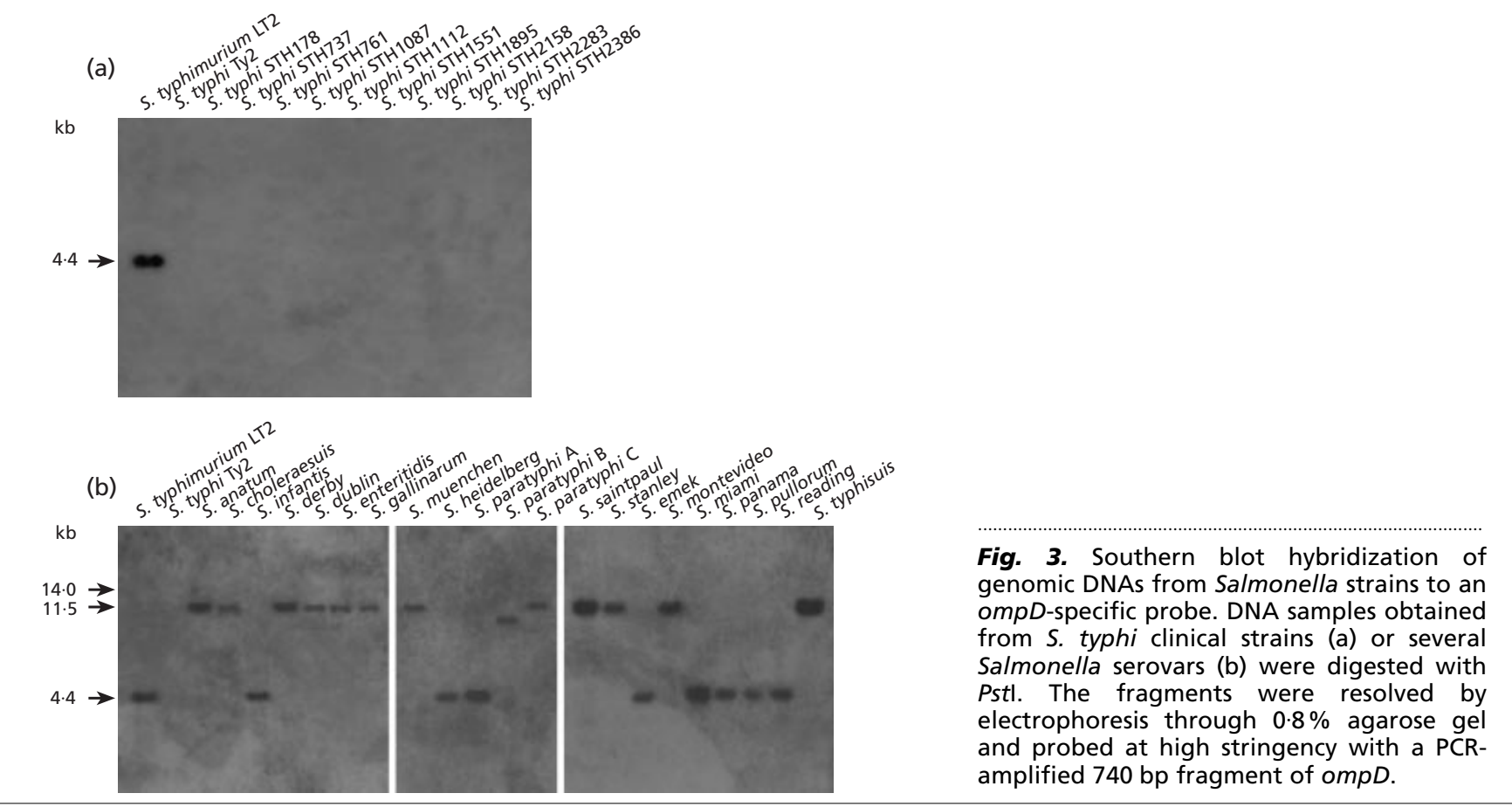

(a)

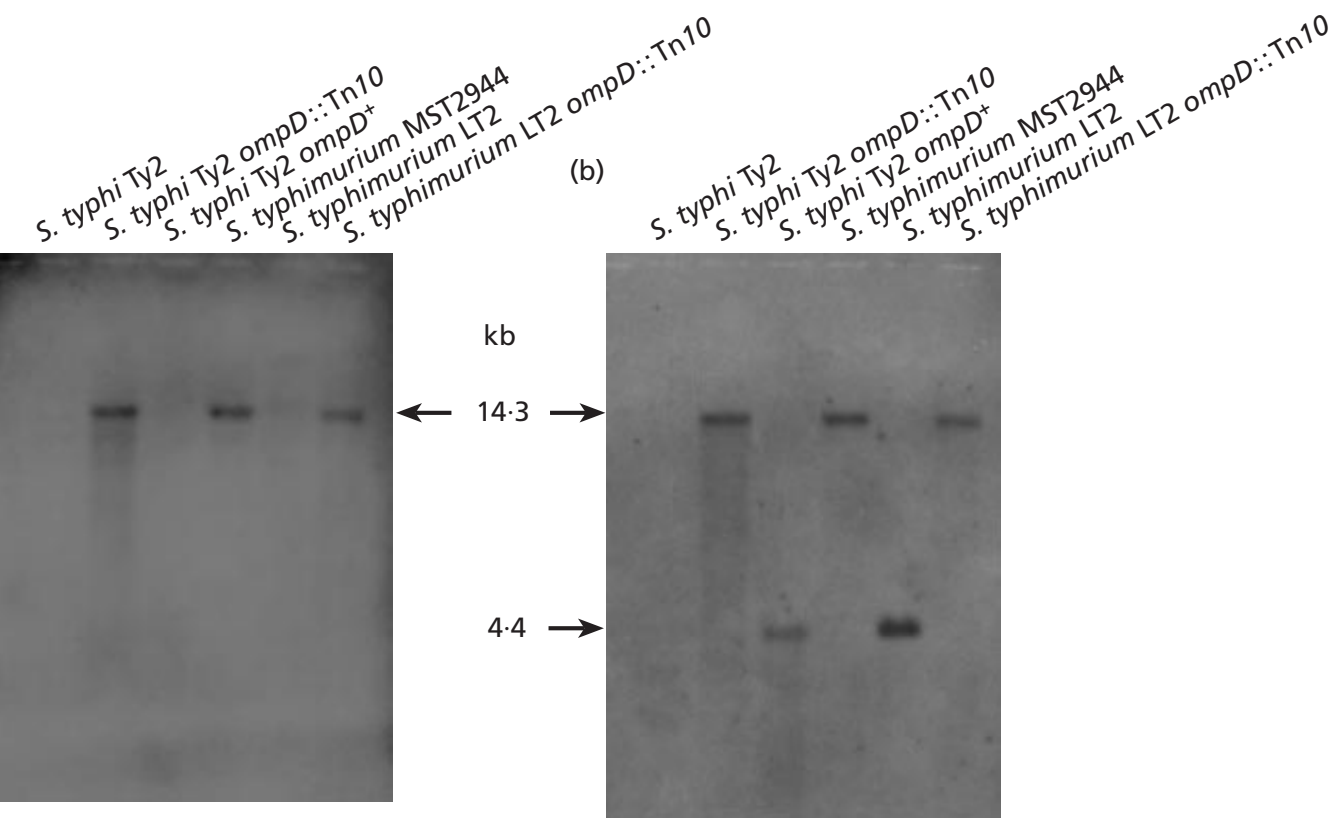

Fig. 4. Southern blot hybridization of genomic DNAs from interspecific hybrids between S. typhimurium and S. typhi. DNA samples were digested with Pstl, fragments were resolved by electrophoresis through $0.8 \%$ agarose gel, then probed at high stringency with a PCR-amplified $740 \mathrm{bp}$ fragment of ompD (b) and with a DNA fragment internal to the Tn10 tetAR genes (a).

typhimurium genome into $S$. typhi by generalized transduction with temperate phage P22 with low but measurable efficiencies. Therefore, we designed a twostep genetic approach using P22-mediated transduction to construct the S. typhi strain Ty2 ompD $D^{+}$.
In a first cross, we introduced an ompD:: Tn10 allele from donor S. typhimurium strain MST2944 into recipient $S$. typhi strain Ty 2 by generalized transduction with phage P22 and selection for recombinants that had acquired resistance to tetracycline. PCR amplification of 


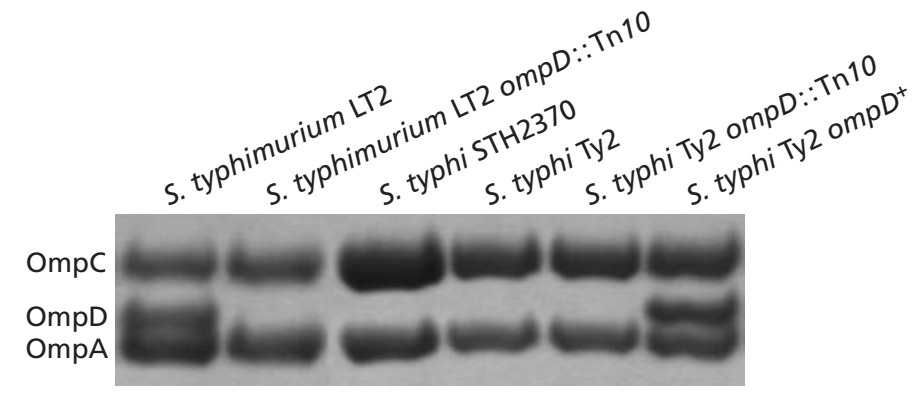

Fig. 5. SDS-PAGE analysis of porins from S. typhimurium and S. typhi interspecific hybrid strains. OMP preparations were obtained from bacteria grown aerobically at $37^{\circ} \mathrm{C}$ in LB broth to stationary phase, resolved by electrophoresis through $12.5 \%$ acrylamide and stained with Coomassie blue. Under the bacterial growth conditions used, OmpF porin is not detected in the OMP profiles.

the ompD gene from $S$. typhimurium strain LT2 ompD ::Tn10 and $S$. typhi strain Ty2 ompD::Tn10 DNA templates yielded the expected $740 \mathrm{bp}$ product, revealing that the amplified fragment of ompD does not include the site of the $\operatorname{mop} D:: \operatorname{Tn} 10$ insertion (Fig. 2a). The presence of the ompD:: Tn10 allele in the chromosome of these interspecific hybrids was confirmed by Southern hybridization, using a probe specific to the tetRA genes of Tn10. As shown in Fig. 4(a), the Tn10 probe hybridizes with a band of approximately $14.3 \mathrm{~kb}$ in PstI-restricted DNA samples obtained from S. typhi strain Ty2 ompD::Tn10 and S. typhimurium strains MST2944 ompD::Tn10 and LT2 ompD::Tn10. A comparison of the OMP profiles by SDS-PAGE clearly showed that $S$. typhimurium strain LT2 ompD:: Tn10 and S. typhi strain Ty2 ompD:: Tn10 do not express the OmpD porin (Fig. 5).

In a second cross, the $S$. typhi strain Ty2 ompD:: Tn10 was used as the recipient of the $o m p D^{+}$allele from $S$. typhimurium strain LT2, selecting for transductants that had lost the tetracycline resistance determinant (Maloy \& Nunn, 1981). PCR amplification of genomic DNA isolated from the $S$. typhi strain Ty 2 ompD $D^{+}$using the ompD-specific primers yielded a $740 \mathrm{bp}$ product (Fig. 2a). Accordingly, a band of approximately $4 \cdot 4 \mathrm{~kb}$ was observed when PstI-digested DNA samples obtained from S. typhi Ty2 ompD $D^{+}$were hybridized with the ompD probe (Fig. $4 \mathrm{~b}$ ). A band of the same size was also observed when S. typhimurium DNA was probed, revealing the presence of the ${ }_{0} m p D^{+}$allele. Remarkably, we confirmed the presence of the OmpD porin in OMP preparations of the S. typhi strain Ty2 ompD $D^{+}$by SDSPAGE (Fig. 5). These results show that the ompD gene can be introduced into the $S$. typhi genome, and can be expressed in this genetic background, suggesting that there are no species-specific factors required for $o m p D$ expression in S. typhi.

\section{S. typhi is highly sensitive to methyl viologen}

The observation that S. typhi strain Ty2, as well as the clinical strains, are missing the $o m p D$ gene is suggestive of a deletion in the $S$. typhi genome with respect to the $S$. typhimurium genome that may extend beyond the ompD gene. Analysis of the S. typhimurium chromosome reveals that $o m p D$ is adjacent to $s m v A$ (Hongo et al., 1994). It has been suggested that SmvA is an innermembrane protein involved in the export of ammonium quaternary substrates, such as methyl viologen, by exporting them to the extracellular space (Hongo et al., 1994). Methyl viologen generates superoxide and oxygen radicals (Farr \& Kogoma, 1991) and has been used in vitro to mimic the oxidative environment found within macrophages (Buchmeier et al., 1997).

Therefore, S. typhi strains, as well as other Salmonella serovars were tested for their sensitivity or resistance to methyl viologen following the same procedure used to identify the S. typhimurium smvA gene (Hongo et al., 1994). Among 35 Salmonella serovars tested, only $S$. typhi strain Ty2 was unable to grow on Luria agar plates supplemented with $0.5 \mathrm{mM}$ methyl viologen. In addition, the 26 clinical isolates of $S$. typhi used in this study were also unable to grow in the presence of the same amount of methyl viologen. To determine the level of resistance to methyl viologen, some strains were tested for their ability to grow in the presence of a range of concentrations of this compound. The highest concentration that allowed the growth of $S$. typhi strain Ty2 and $S$. typhimurium strain LT2 were $0.4 \mathrm{mM}$ and 50 $\mathrm{mM}$, respectively. These results suggest either that $S$. typhi is missing the $s m v A$ gene, or that the $s m v A$ gene is not functional in this serovar.

To distinguish between these two alternatives, primers from the reported smvA sequence (GenBank accession no. D26057) were designed to amplify a portion of this gene. Amplification of a $S$. typhi strain Ty2 DNA template did not yield a product internal to smvA. In contrast, amplification of chromosomal DNA isolated from $S$. typhimurium strains LT2 ompD ${ }^{+}$and LT2 ompD::Tn10, as well as $S$. typhi strains Ty2 ompD:: Tn10 and Ty 2 ompD $D^{+}$, resulted in the expected 940 bp product (Fig. 6a). Remarkably, DNA obtained from four randomly chosen clinical isolates of S. typhi, which were sensitive to methyl viologen, yielded an amplification product of the expected size (Fig. 6b). Thus, although the SmvA function is absent from all tested $S$. typhi strains, the $s m v A$ gene appears to be present in $S$. typhi clinical strains.

In addition, the 940 bp smvA fragment from S. typhimurium was labelled and used to probe Southern blots of $S$. typhi DNA digested with PstI. Since there is a single $P s t \mathrm{I}$ site within the $S$. typhimurium smvA sequence reported, and this site is present in the probe, the $s m v A$ probe hybridized with two fragments (approx. 4.4 and $11.8 \mathrm{~kb}$ ) present in S. typhimurium strain LT2 DNA 


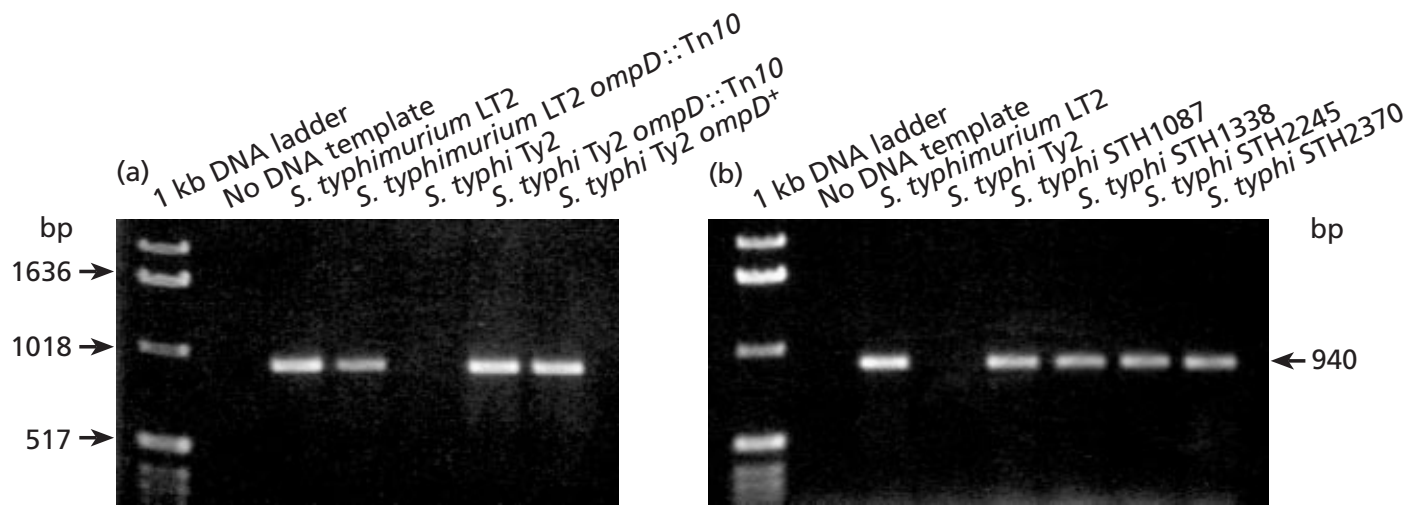

Fig. 6. Amplification of the smvA gene from Salmonella strains. PCR amplifications were performed as described in Methods, using primers internal to smvA. Chromosomal DNA templates were obtained from (a) interspecific hybrids between S. typhimurium and S. typhi, and (b) S. typhi clinical strains. RFLP analysis indicated that the smvA fragment amplified from S. typhi clinical strains does not have the Pstl site present in the S. typhimurium smvA gene (unpublished observation).

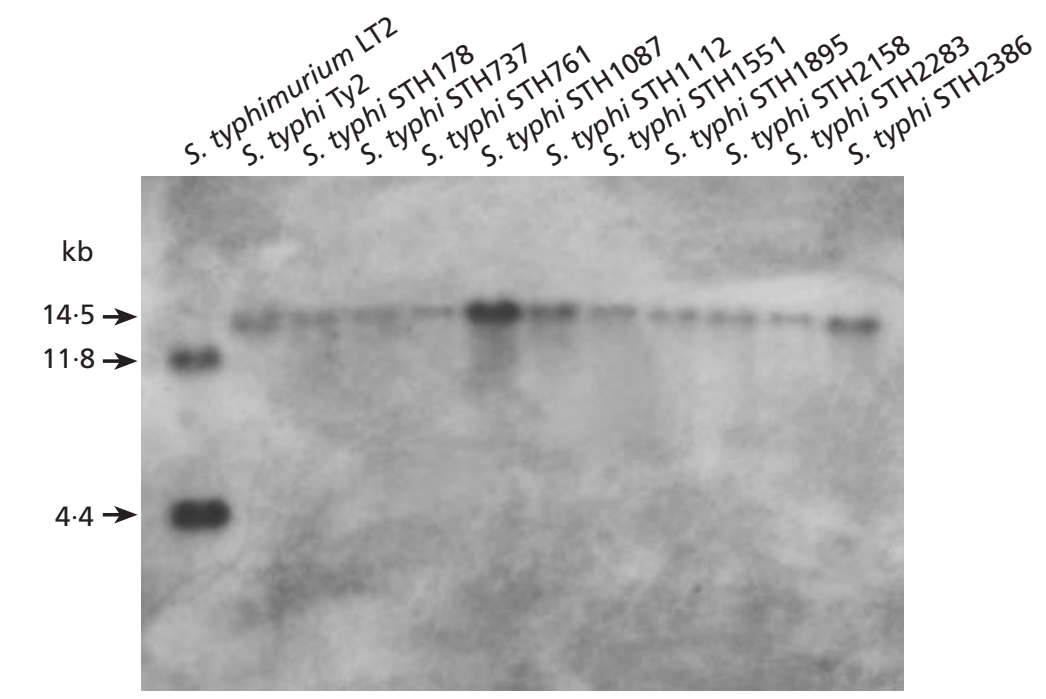

Fig. 7. Southern blot hybridization of genomic DNAs from Salmonella strains to a smvA-specific probe. DNA samples were digested with Pstl. The fragments were resolved by electrophoresis through $0.8 \%$ agarose gel and probed at high stringency with a PCR-amplified $940 \mathrm{bp}$ fragment of smvA. Only one band was observed when DNA isolated from $S$. typhi was probed because $S$. typhi smvA does not have the Pstl site present in the $S$. typhimurium smvA gene.

(Fig. 7). The smaller band represents a fragment containing both $o m p D$ and a portion of $s m v A$, because it also hybridized with the ompD probe. On the other hand, in contrast to the PCR results suggesting that $s m v A$ is missing from $S$. typhi strain $\mathrm{Ty} 2$, the specific $s m v A$ probe hybridized with a single band of approximately $14.5 \mathrm{~kb}$ in DNA samples obtained from this strain (Fig. 7). The same hybridization band was observed when DNA isolated from 10 randomly chosen clinical isolates of S. typhi was probed (Fig. 7). This is because $S$. typhi smvA does not have the PstI site present in the $S$. typhimurium smvA gene, as indicated by RFLP analysis (data not shown).

\section{DISCUSSION}

The OmpD porin is one of the most abundant proteins in the outer membrane of many different Salmonella serovars, with the exception of S. typhi. Our results demonstrate that, due to the absence of the ompD gene,
S. typhi does not produce the OmpD porin. Both PCR and Southern analyses show that the OmpD coding sequence is absent from the $S$. typhi genome. Nonetheless, it was possible to construct a derivative of $S$. typhi carrying the $S$. typhimurium ompD gene which expressed the porin. Presumably, this interspecific hybrid carries only a small cassette of genes that includes $o m p D$, and provides us with a control suggesting that there are no species-specific factors required for ompD expression in S. typhi.

To construct a recombinant strain with a single copy of ompD in the proper genetic position, we introduced an ompD::Tn10 allele from S. typhimurium MST2944 donor strain into recipient $S$. typhi strain $\mathrm{Ty} 2$ by generalized transduction with $S$. typhimurium phage P22. This transduction was originally performed by Liu \& Sanderson (1995a, b) as one of a systematic set of transductions used to assign genes on the S. typhi strain Ty 2 genome to positions corresponding to those on the S. typhimurium genetic map. Using this approach, Liu 
\& Sanderson (1995a, b) concluded that the ompD gene is located in the centre of a $500 \mathrm{~kb}$ inversion which includes the replication terminus of the $S$. typhi strain Ty2 chromosome. The ompD:: $\operatorname{Tn} 10$ allele from $S$. typhimurium can be transduced into the S. typhi genome even though the recipient does not have the corresponding homeologous gene. This is because P22 packages about $44 \mathrm{~kb}$ of DNA, and P22-mediated generalized transduction permits homologous recombination events to occur in genes flanking a region of DNA that is not present in the recipient genome.

Our Southern blot analyses show that all S. typhi strains tested retain an $s m v A$ coding sequence, but they are nevertheless sensitive to methyl viologen, suggesting that the gene is not expressed in these strains. Alternatively, they may be lacking or be defective in additional genes required for methyl viologen resistance. In Escherichia coli, a related Gram-negative bacillus, at least three genes dispersed on the bacterial genome are required for methyl viologen resistance, and mutations in each of these genes confer sensitivity to methyl viologen (Morymio, 1988; Morymio et al., 1992). Transductants of $S$. typhi strain Ty2 carrying the ompD $D^{+}$ allele and the cotransduced $s m v A$ gene express the OmpD porin in the outer membrane, and acquire resistance to methyl viologen (unpublished observation), suggesting that the $s m v A$ gene in clinical isolates of $S$. typhi may not be functional. Thus, the ompD-smvA region is genetically variable among different Salmonella serovars.

The ompD and $s m v A$ genes have been mapped at centisomes 33.7 and 38.6 , respectively, in the S. typhimurium chromosome (Sanderson et al., 1995). Because both genes are cotransducible with phage P22, they cannot be that far apart. Instead, our results confirm those of Singh et al. (1996) that place three S. typhimurium genes, ompD, smvA and narU, at centisome 33.7 (GenBank accession no. D26057). Analysis of the $S$. typhi genome sequence data released by the Sanger Center (http://www.sanger.ac.uk/Projects/S_typhi/ BLAST_server.shtml and ftp://ftp.sanger.ac.uk/pub/ pathogens/st/ST.dbs) reveals that S. typhi has two nar operons, $85 \%$ and $81 \%$ identical at the nucleotide level to the E. coli narGHJI and narZYWV operons, respectively. Both nar operons are also present in $S$. typhimurium (Barrett \& Riggs, 1982; Spector et al., 1999), but only one of them (narGHIJ) has been mapped, lying at centisome 38.6 (Casse et al., 1973; Sanderson et al., 1995). In E. coli, the narU gene is located immediately upstream of the narZYWV operon (Gennis \& Stewart, 1996). Given the remarkable conservation of gene order between E. coli strain K12 and S. typhimurium strain LT2 (Krawiec \& Riley, 1990), we can assign the narZYWV operon to a map position at centisome $33 \cdot 7$ on the $S$. typhimurium genome, adjacent to the ompD$-s m v A-n a r U$ region. Recently, the Genome Sequence Center from the Washington University School of Medicine (http://genome.wustl.edu/gsc/Projects/bacterial/salmonella.shtml) sequenced an ORF upstream of the S. typhimurium ompD gene. This ORF, designated $y d d G$, encodes a putative transmembrane permease. Notably, $y d d G$ is not present in the S. typhi genome sequence data released by the Sanger Center, suggesting that the genetic variability in the ompD-smvA region of the Salmonella genome also includes the $y d d G$ locus.

Because S. typhi is the only Salmonella serovar missing the $\operatorname{ompD}_{\mathrm{D}}$ gene, the determination of its presence in other Salmonella serovars has a clinical significance. This kind of assay allows us to distinguish S. typhi from $S$. paratyphi serovars that cause systemic human infections presenting nearly identical symptoms. Thus, a PCR assay could be developed by using primers hybridizing upstream and downstream of the presumed deletion in the S. typhi strains, where the length of the amplified segment should discriminate between the different Salmonella serovars responsible for clusters of typhoid-like fevers and thus allow a more accurate assessment of their epidemiology.

What is the role of the ompD gene in Salmonella infection? The $o m p D$ gene does not appear to play a major role in the virulence of Salmonella serovars, because the effects of mutations in the ompD gene on the virulence of S. typhimurium are subtle. Dorman et al. (1989) found that a strain with a Tn10 insertion in ompD has a slightly greater $\mathrm{LD}_{50}$ than its otherwise isogenic wild-type parent. However, Meyer et al. (1998) found that there was no statistically significant difference between the $\mathrm{LD}_{50}$ of $S$. typhimurium wild-type and ompD mutant strains.

Although it seems likely that the ompD gene plays only a minor role in virulence, it may be important for determining host specificity. Many studies have characterized the genetic determinants of Salmonella virulence; however, very little is known about the genetic determinants of host specificity. Initial attempts to define the genetic basis of host specificity revealed that its determinants in Salmonella are likely to be multifactorial. In generalized transduction experiments with phage P22 grown in a $S$. typhimurium donor and with $S$. typhi recipients, no virulent recombinants that could kill mice were obtained (Zahrt, 1998). These negative results indicate that multiple genetic differences between these serovars must contribute to host specificity.

We hypothesize that the ompD-smvA region contributes to host specificity, because the presence of $o m p D$ is strongly correlated with the ability of Salmonella serovars to grow in alternative, non-human hosts. OmpD is present in all Salmonella serovars, such as $S$. typhimurium and $S$. enteritidis, that grow in multiple mammalian hosts. In contrast, $\mathrm{OmpD}$ is absent from $S$. typhi, which can grow only in a human host. Although the $S$. paratyphi $\mathrm{A}, \mathrm{B}$ and $\mathrm{C}$ serovars express $\mathrm{OmpD}$, and were once thought to cause systemic infections only in humans, several reports have shown that S. paratyphi serovars $\mathrm{B}$ and $\mathrm{C}$ are not restricted to a human host and can infect cattle and poultry (George et al., 1972; Thomas, 1978; Ojeniyi, 1984). The almost perfect correlation between the absence of $o m p D$ and a host range restricted to humans raises the important question 
of whether S. paratyphi A also has an alternative vertebrate host.

In recent years, it has become clear that the outermembrane porins are involved in the interactions of Gram-negative species, including Helicobacter pylori (Tufano et al., 1994), Neisseria gonorrhoeae (Bauer et al., 1999), Shigella flexneri (Bernardini et al., 1993), S. typhi (Blanco et al., 1997) and possibly Pasteurella haemolytica (Davies et al., 1997), with their specific hosts. The OmpD porin must play a critical role in the survival of S. typhimurium in certain environments, because OmpD facilitates the entry of some critical nutrients (Nikaido, 1996). Furthermore, OmpD may also play a critical role in the interactions between $S$. typhimurium and host cells, and is likely to be involved in the adhesion of S. typhimurium to murine macrophages (Negm \& Pistole, 1998).

The characterization of this important genomic difference between S. typhi and other Salmonella serovars that includes $o m p D$ is significant for the following three reasons. First, the report of similar small differences in genome structure among different serovars will enable the development of additional PCR assays for a more robust assessment of the epidemiology of Salmonella outbreaks. Second, by demonstrating that generalized transduction can be used to construct interspecific hybrids between different Salmonella serovars, it is possible to take a genetic approach to assessment of the contributions of small genomic differences between two serovars to differences in host specificity. Third, the correlation between the presence of the ompD gene and the ability of Salmonella serovars to infect vertebrate, non-human hosts is the first reported correlation between an individual gene and the host range of Salmonella serovars.

\section{ACKNOWLEDGEMENTS}

We are indebted to Philip Youderian and Alexis Kalergis for encouragement and critical reading of this manuscript. We thank Stanley Maloy and Ana María Cordano for generous gifts of bacterial strains. This work was supported by grants 1960255 and 1990153 from FONDECYT. C.A.S. was supported by a fellowship from CONICYT.

\section{REFERENCES}

Anderson, R. P. \& Roth, J. R. (1981). Spontaneous tandem genetic duplications in Salmonella typhimurium arise by unequal recombination between ribosomal RNA (rrn) cistrons. Proc Natl Acad Sci U S A 78, 3113-3117.

Barrett, E. L. \& Riggs, D. L. (1982). Evidence for a second nitrate reductase activity that is distinct from the respiratory enzyme in Salmonella typhimurium. J Bacteriol 150, 563-571.

Bauer, F. J., Rudel, T., Stein, M. \& Meyer, T. F. (1999). Mutagenesis of the Neisseria gonorrhoeae porin reduces invasion in epithelial cells and enhances phagocyte responsiveness. Mol Microbiol 31, 903-913.

Bäumler, A. J., Tsolis, R. M., Ficht, T. A. \& Adams, L. G. (1998). Evolution of host adaptation in Salmonella enterica. Infect Immun 66, 4579-4587.

Bernardini, M. L., Sanna, M. G., Fontaine, A. \& Sansonetti, P. J.
(1993). OmpC is involved in invasion of epithelial cells by Shigella flexneri. Infect Immun 61, 3625-3635.

Blanco, L. P., Toro, C. S., Romero, J. M., Santiviago, C. A. \& Mora, G. C. (1997). Salmonella typhi Ty2 OmpC porin induces bactericidal activity on U937 monocytes. Microbiol Immunol 40, 999-1004.

Buchmeier, N., Bossie, S., Chen, C. Y., Fang, F. C., Guiney, D. G. \& Libby, S. J. (1997). Sly, a transcriptional regulator of Salmonella typhimurium, is required for resistance to oxidative stress and is expressed in the intracellular environment of macrophages. Infect Immun 65, 3725-3730.

Calderón, I., Lobos, S. R. \& Mora, G. C. (1984). The hemolytic effect of Salmonella typhi Ty2 porins. Eur J Biochem 141, 579-583.

Casse, F., Chippaux, M. \& Pascal, M. C. (1973). Isolation from Salmonella typhimurium LT2 of mutants lacking specifically nitrate reductase activity and mapping of the chlC gene. Mol Gen Genet 124, 247-251.

Davies, R. L., Arkinsaw, S. \& Selander, R. K. (1997). Evolutionary genetics of Pasteurella haemolytica isolates recovered from cattle and sheep. Infect Immun 65, 3585-3593.

Dorman, C. J., Chatfield, S., Higgins, C. F., Hayward, C. \& Dougan, G. (1989). Characterization of porin and $o m p R$ mutants of a virulent strain of Salmonella typhimurium: ompR mutants are attenuated in vivo. Infect Immun 57, 2136-2140.

Echeita, M. A. \& Usera, M. A. (1998). Chromosomal rearrangements in Salmonella enterica serotype Typhi affecting molecular typing in outbreak investigations. J Clin Microbiol 36, 2123-2126.

Farr, S. B. \& Kogoma, T. (1991). Oxidative stress responses in Escherichia coli and Salmonella typhimurium. Microbiol Rev 55, 561-585.

Gennis, R. B. \& Stewart, V. (1996). Respiration. In Escherichia coli and Salmonella: Cellular and Molecular Biology, 2nd edn, vol. 1, pp. 217-261. Edited by F. C. Neidhardt and others. Washington, DC: American Society for Microbiology.

George, J. T., Wallace, J. G., Morrison, H. R. \& Harbourne, J. F. (1972). Paratyphoid in man and cattle. Br Med J 3, 208-211.

Hongo, E., Morimyo, M., Mita, K., Machida, I., Hama-Inaba, H., Tsuji, H., Ichimura, S. \& Noda, Y. (1994). The methyl viologenresistance-encoding gene $s m v A$ of Salmonella typhimurium. Gene 148, 173-174.

Kleckner, N., Bender, J. \& Gottesman, S. (1991). Uses of transposons with emphasis on Tn10. Methods Enzymol 204, 139-180.

Krawiec, S. \& Riley, M. (1990). Organization of the bacterial chromosome. Microbiol Rev 54, 502-539.

Lan, R. T. \& Reeves, P. R. (1996). Gene transfer is a major factor in bacterial evolution. Mol Biol Evol 13, 47-55.

Lee, D. \& Schnaitman, C. (1980). Comparison of outer membrane proteins produced by Escherichia coli and Salmonella typhimurium. J Bacteriol 142, 1019-1022.

Liu, S.-L. \& Sanderson, K. E. (1995a). Genomic cleavage map of Salmonella typhi Ty2. J Bacteriol 177, 5099-5107.

Liu, S.-L. \& Sanderson, K. E. (1995b). Rearrangements in the genome of the bacterium Salmonella typhi. Proc Natl Acad Sci US A 92, 1018-1022.

Liu, S.-L. \& Sanderson, K. E. (1996). Highly plastic chromosomal organization in Salmonella typhi. Proc Natl Acad Sci US A 93, 10303-10308.

Lobos, S. R. \& Mora, G. C. (1991). Alterations in the electrophoretic mobility of OmpC due to variations in the am- 
monium persulfate concentration in sodium dodecylsulfatepolyacrylamide gel electrophoresis. Electrophoresis 12, 448-450.

McClelland, M. \& Wilson, R. K. (1998). Comparison of sample sequences of the Salmonella typhi genome to the sequence of the complete Escherichia coli K-12 genome. Infect Immun 66, 4305-4312.

Maloy, S. R. (1990). Experimental Techniques in Bacterial Genetics. Boston, MA: Jones \& Bartlett.

Maloy, S. R. \& Nunn, W. (1981). Selection for loss of tetracycline resistance by Escherichia coli. J Bacteriol 145, 1110-1112.

Meyer, P. N., Wilmes-Riesenberg, M. R., Stathopoulos, C. \& Curtiss, R. (1998). Virulence of a Salmonella typhimurium OmpD mutant. Infect Immun 66, 387-390.

Morymio, M. (1988). Isolation and characterization of methyl viologen-sensitive mutants of Escherichia coli K-12. J Bacteriol 170, 2136-2142.

Morymio, M., Hongo, E., Hama-Inaba, H. \& Machida, I. (1992). Cloning and characterization of the murC gene of Escherichia coli K-12 which confers resistance against methyl viologen toxicity. Nucleic Acids Res 20, 3159-3165.

Negm, R. S. \& Pistole, T. G. (1998). Macrophages recognize and adhere to an OmpD-like protein of Salmonella typhimurium. FEMS Immunol Med Microbiol 20, 191-199.

Ng, I., Liu, S.-L. \& Sanderson, K. E. (1999). Role of genomic rearrangement in producing new ribotypes of Salmonella typhi. J Bacteriol 181, 3536-3541.

Nikaido, H. (1996). Outer membrane. In Escherichia coli and Salmonella: Cellular and Molecular Biology, 2nd edn, vol. 1, pp. 29-47. Edited by F. C. Neidhardt and others. Washington, DC: American Society for Microbiology.

Nikaido, H. \& Vaara, M. (1985). Molecular basis of bacterial outer membrane permeability. Microbiol Rev 49, 1-32.

Ojeniyi, A. (1984). 'Salmonella hirschfeldii' in poultry and man in Ibadan, Nigeria. Bull World Health Organ 62, 773-775.

Sambrook, J., Fritsch, E. F. \& Maniatis, T. (1989). Molecular Cloning: a Laboratory Manual, 2nd edn. Cold Spring Harbor, NY: Cold Spring Harbor Laboratory.
Sanderson, K. E., Hessel, A. \& Rudd, K. (1995). Genetic map of Salmonella typhimurium, Edition VIII. Microbiol Rev 59, 241-303.

Schnaitman, C. A. (1971). Solubilization of the cytoplasmic membrane of Escherichia coli by Triton X-100. J Bacteriol 108, $545-552$.

Selander, R., Li, J. \& Nelson, K. (1996). Evolutionary genetics of Salmonella enterica. In Escherichia coli and Salmonella: Cellular and Molecular biology, 2nd edn, vol. 2, pp. 2691-2707. Edited by F. C. Neidhardt and others. Washington, DC: American Society for Microbiology.

Singh, S. P., Miller, S., Williams, Y. U., Rudd, K. E. \& Nikaido, H. (1996). Immunochemical structure of the OmpD porin for Salmonella typhimurium. Microbiology 142, 3201-3210.

Spector, M. P., Garcia del Portillo, F., Bearson, S. M. D., Mahmud, A., Magut, M., Finlay, B. B., Dougan, G., Foster, J. W. \& Pallen, M. J. (1999). The $r p o S$-dependent starvation-stress response locus stiA encodes a nitrate reductase (narZYWV) required for carbonstarvation-inducible thermotolerance and acid tolerance in Salmonella typhimurium. Microbiology 145, 3035-3045.

Thomas, G. W. (1978). Salmonella paratyphi B in cattle. Vet Rec 103, 512.

Tufano, M. A., Rossano, F., Catalanotti, P., Liguori, G., Capasso, C., Ceccarelli, M. T. \& Marinelli, P. (1994). Immunobiological activities of Helicobacter pylori porins. Infect Immun 62, 1392-1399.

Zahrt, T. C. (1998). A genetic analysis of the Salmonella typhi host range. $\mathrm{PhD}$ thesis, University of Illinois.

Zahrt, T. C. \& Maloy, S. (1997). Barriers to recombination between closely related bacteria: MutS and RecBCD inhibit recombination between Salmonella typhimurium and Salmonella typhi. Proc Natl Acad Sci U S A 94, 9786-9791.

Zahrt, T. C., Mora, G. C. \& Maloy, S. (1994). Inactivation of mismatch repair overcomes the barrier to transduction between Salmonella typhimurium and Salmonella typhi. J Bacteriol 176, 1527-1529.

Received 27 February 2001; accepted 26 March 2001. 